Check for updates

Cite this: J. Mater. Chem. B, 2020, 8,5234

Received 3rd April 2020,

Accepted 12th May 2020

DOI: $10.1039 / \mathrm{d} 0 \mathrm{tb} 00888 \mathrm{e}$

rsc.li/materials-b

\section{Pyridinium-substituted tetraphenylethylene salt-based photosensitizers by varying counter anions: a highly efficient photodynamic therapy for cancer cell ablation and bacterial inactivation $\dagger$}

\author{
Wei Xiong, ${ }^{a}$ Lingyun Wang, (D) *a Xiaoli Chen, ${ }^{a}$ Hao Tang, (D) a Derong Cao, (D) ${ }^{a}$ \\ Guozhen Zhang (D) ${ }^{* b}$ and Wei Chen (D) *c
}

\begin{abstract}
Cancer and bacterial infection seriously threaten the health of human beings. The development of an image-guided photosensitizer with a "Two-in-One" function that can be simultaneously used for both efficient cancer cell ablation and rapid bacterial inactivation is highly in demand. In this project, we designed and prepared two aggregation-induced emission luminogens (AIEgens) (called TPEPy-I and TPEPy-PF6) with a strong electron push-pull effect. They have a near-infrared (NIR) emission, a high ${ }^{1} \mathrm{O}_{2}$ quantum yield up to 0.93 and a fluorescence turn-on effect in mitochondria. Upon white light irradiation, the two mitochondria-targeting AlEgens exhibit a highly efficient photodynamic ablation of HeLa cells as well as excellent photodynamic inactivation of both Gram-positive S. aureus and Gramnegative $E$. coli. The time-dependent density functional theory (TD-DFT) results indicate that compared to TPEPy-PF6, TPEPy-I can easily produce the triplet state that is a prerequisite for ${ }^{1} \mathrm{O}_{2}$ formation. Moreover, the positive effect of iodide anions gives TPEPy-I a higher photodynamic efficacy in cancer cell ablation and bacterial inactivation as compared with TPEPy-PF6.
\end{abstract}

\section{Introduction}

Cancer and bacterial infectious diseases threaten human health. Especially, cancer-associated bacteria seriously reduce the efficiency of cancer treatments. ${ }^{1}$ It is important and urgent to develop an effective method enabling cancer therapy and killing of pathogenic bacteria simultaneously. Photodynamic therapy (PDT) has emerged as a promising method to combat cancer and pathogenic bacteria due to its distinct advantages, such as noninvasiveness, negligible drug resistance, localized treatment, high spatiotemporal precision and low side effects. ${ }^{2}$ For instance, Photofrin is the first FDAapproved photosensitizer (PS) and is still widely employed in clinical cancer treatment. Its mechanism is associated with the generation of destructive singlet oxygen $\left({ }^{1} \mathrm{O}_{2}\right)$ or other reactive oxygen species (ROS) under light irradiation. ${ }^{3}$ Specifically, using

\footnotetext{
${ }^{a}$ School of Chemistry and Chemical Engineering, South China University of Technology, Guangzhou, 510640, China. E-mail: lingyun@scut.edu.cn

${ }^{b}$ Hefei National Laboratory for Physical Sciences at the Microscale, Collaborative Innovation Center of Chemistry for Energy Materials, CAS Center for Excellence in Nanoscience, School of Chemistry and Materials Science, University of Science and Technology of China, Hefei, Anhui 230026, P. R. China.

E-mail: guozhen@ustc.edu.cn

${ }^{c}$ Department of Physics, The University of Texas at Arlington, Arlington, Texas 76019, USA. E-mail: weichen@uta.edu

$\dagger$ Electronic supplementary information (ESI) available. See DOI: 10.1039/d0tb00888e
}

an emissive PS to realize image-guided PDT is important and attractive, since it conveniently offers diagnosis and treatment integration. However, traditional PSs suffer from quenched fluorescence and reduced ROS production in the aggregated state because of $\pi-\pi$ stacking and the resulting aggregationcaused quenching (ACQ) effect, which makes the image-guided PDT unsatisfactory.

In contrast, aggregation-induced-emission PSs (AIE-PSs) show enhanced fluorescence and efficient photosensitizing characteristics in aggregate states, which can avoid the ACQ effect of PSs. ${ }^{4-16}$ In addition, some groups revealed that AIE luminogens (AIEgens) with an electron push-pull effect would favor ${ }^{1} \mathrm{O}_{2}$ generation. ${ }^{17}$ To date, AIE-PSs have been widely employed in imaging-guided cancer cell ablation, and bacterial detection and inactivation. $^{18-20}$ However, some AIE-PSs are ineffective in PDT mediated killing of Gram-negative bacteria. $^{20 f g}$ Particularly in solution, Gram-negative bacteria are protected from extracellularly produced singlet oxygen. ${ }^{21}$ More importantly, porins at the outer membranes of Gram-negative bacteria can work as 'molecular sieves', which hinder PSs of large size from going through the porin channels. These synergistic effects make PSs fail to kill Gramnegative species. Furthermore, bacterial infection as a cause of cancer has been studied, where the induction of chronic inflammation and the production of carcinogenic bacterial metabolites are regarded as possible mechanisms. ${ }^{22}$ The most 


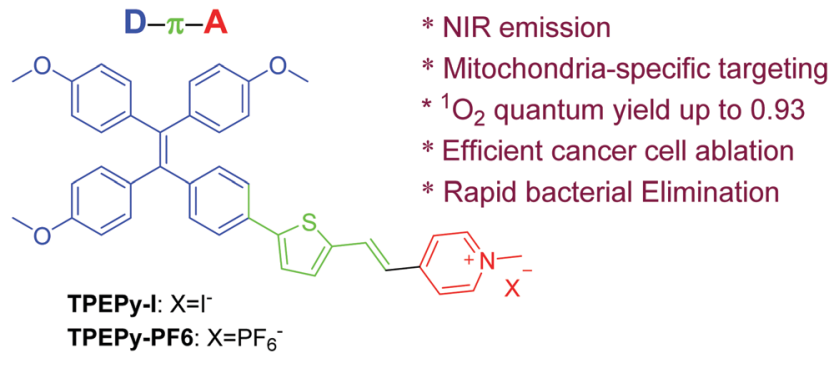

Scheme 1 Synthetic routes to TPEPy-I and TPEPy-PF6.

specific example of the inflammatory mechanism of carcinogenesis is Helicobacter pylori infection. In addition, skin cancers are easily induced by bacterial infections. Therefore, the development of efficient AIE-PSs that can simultaneously kill cancer cells and bacteria would be appealing.

Recently, Chen's group reported a powerful combination of copper-cysteamine nanoparticles with potassium iodide to inactivate both Gram-positive MRSA and Gram-negative E. coli. ${ }^{23}$ In that case, iodide anions showed a positive effect by the formation of triiodide ions to enhance the bactericidal ability. Inspired by this, a cationic AIE-PS containing an iodide anion (TPEPy-I, Scheme 1) was designed and synthesized by a simple and straightforward synthetic protocol. For comparison, another AIEPS containing hexafluorophosphate (TPEPy-PF6, Scheme 1) was introduced for the same study. Both AIE-PSs have some interesting features, such as: (1) the AIEgens comprise a TPE segment (working as a donor), a thiophene fragment ( $\pi$ bridge), and a cationic pyridinium moiety (acceptor), enabling a broad absorption in the visible range and ${ }^{1} \mathrm{O}_{2}$ formation through a strong charge transfer. (2) Mitochondrion is an important site for energy conversion and the main source of cellular ROS, which plays a crucial role in mediating cell apoptosis. The presence of cationic pyridinium endows the two AIEgens with a function of targeting mitochondria that may improve PDT performance and imaging qualities. Meanwhile, the cationic pyridinium may help AIEgens penetrate the bacterial membranes by electrostatic interaction and improve their water solubility. (3) The D- $\pi-\mathrm{A}$ structure endows AIE-PSs with NIR emissions, which is highly desirable to achieve image-guided PDT with a low background noise. Our observations indicate that both TPEPy-I and TPEPy-PF6 have the "two in one" functions that can be used simultaneously for cancer cell ablation and bacterial inactivation. However, TPEPy-I is more efficient as an AIE-PS than TPEPy-PF6, in which the iodide ions may play some important roles in the photodynamic performance.

\section{Results and discussion}

\subsection{Molecular design and synthesis}

As shown in Scheme S1 (ESI $\dagger$ ), compound 2 was prepared by a Suzuki reaction between TPE derivative 1 and 5-formyl-2thiopheneboronic acid. TPEPy-I was obtained by the Knoevenagel reaction between 2 and pyridinium salt. The anion exchange between TPEPy-I and hexafluorophosphate generated TPEPy-PF6, which was expected to avoid the fluorescence quenching effect of iodine atoms. The combination of a strong electron donoracceptor (D-A) interaction with extended $\pi$-conjugation could facilitate intramolecular charge transfer (ICT), therefore resulting in low electronic bandgaps, broad absorption and long emission wavelengths. The two target products were characterized by NMR and HRMS (see Fig. S1-S6 in the ESI $\dagger$ for the details).

\subsection{Solvatochromism and AIE properties}

As shown in Fig. S7 and S8 (ESI $\dagger$ ), TPEPy-I and TPEPy-PF6 show obvious solvatochromism, where their absorption and emission spectra are remarkably affected by the polarity of solvents. For example, TPEPy-I in THF has a broad absorption from 350 to $550 \mathrm{~nm}$ with the absorption maximum at $453 \mathrm{~nm}$ (with a $40000 \mathrm{M}^{-1} \mathrm{~cm}^{-1}$ molar extinction coefficient) and an emission at $744 \mathrm{~nm}$ with a large Stokes shift of $191 \mathrm{~nm}$. Similarly, TPEPy-PF6 in THF show a large Stokes shift of $169 \mathrm{~nm}$ with a maximum absorption at $464 \mathrm{~nm}$ (with a $44000 \mathrm{M}^{-1} \mathrm{~cm}^{-1}$ molar extinction coefficient) and an emission maximum at $733 \mathrm{~nm}$. In addition, the emission intensity of the two AIEgens is greatly reduced or even quenched in polar solvents such as DMSO and DMF, suggesting a strong ICT effect in polar media. As compared with TPEPy-PF6, the heavy atom effect of TPEPy-I slightly weakens the emission intensity. The Stokes shift for TPEPy-I and TPEPy-PF6 is much larger than that of the commercial NIR fluorophores (less than $50 \mathrm{~nm}){ }^{19}$ which can avoid light interference from the excitation light and the self-absorption of emission during biomedical imaging.

The investigation of the AIE feature in DMSO/water mixtures with different water fractions was carried out and it was found that their emissions were almost totally quenched in DMSO. Their photoluminescence (PL) intensities are gradually enhanced with increasing the fraction of water (Fig. S9, ESI $\dagger$ ). The strongest PL intensity was observed at a 90\% fraction of water, in which the PL intensities of TPEPy-I and TPEPy-PF6 were enhanced to about 6.5- and 5.9-fold, respectively, as compared with that of the DMSO solutions. DLS revealed that the average hydrodynamic diameters of nanoaggregates that formed in DMSO/water $(1 / 9, \mathrm{v} / \mathrm{v})$ are around 179 and $301 \mathrm{~nm}$ for TPEPy-I and TPEPy-PF6, respectively (Fig. S10, ESI $\dagger$ ). Meanwhile, TPEPy-I and TPEPy-PF6 also have strong fluorescence in the solid state, and their powder samples show emission peaks at 720 and $718 \mathrm{~nm}$, respectively, (Fig. S11a, ESI $\dagger$ ). The emission spectra of TPEPy-I and TPEPy-PF6 solids in PMMA films are shown in Fig. S11b (ESI $\dagger$ ) and their absolute PL quantum yields were found to be $16 \%$ and $45 \%$, respectively. All these results demonstrate that both TPEPy-I and TPEPy-PF6 are typical AIE-active molecules.

\subsection{ROS and singlet oxygen generation}

Because the two AIEgens have a strong absorption in the visible light region, they can be activated by visible light for PDT. The ROS generation was evaluated under white light excitation using a commercial ROS indicator, $2^{\prime}, 7^{\prime}$-dichlorodihydrofluorescein diacetate $\left(\mathrm{H}_{2}\right.$ DCF-DA), which is capable of emitting a green fluorescence at around $525 \mathrm{~nm}$ when oxidized by ROS. As shown in Fig. 1, for the mixture of each AIEgen and $\mathrm{H}_{2}$ DCF-DA in aqueous solution, the emission at $525 \mathrm{~nm}$ was gradually intensified with 

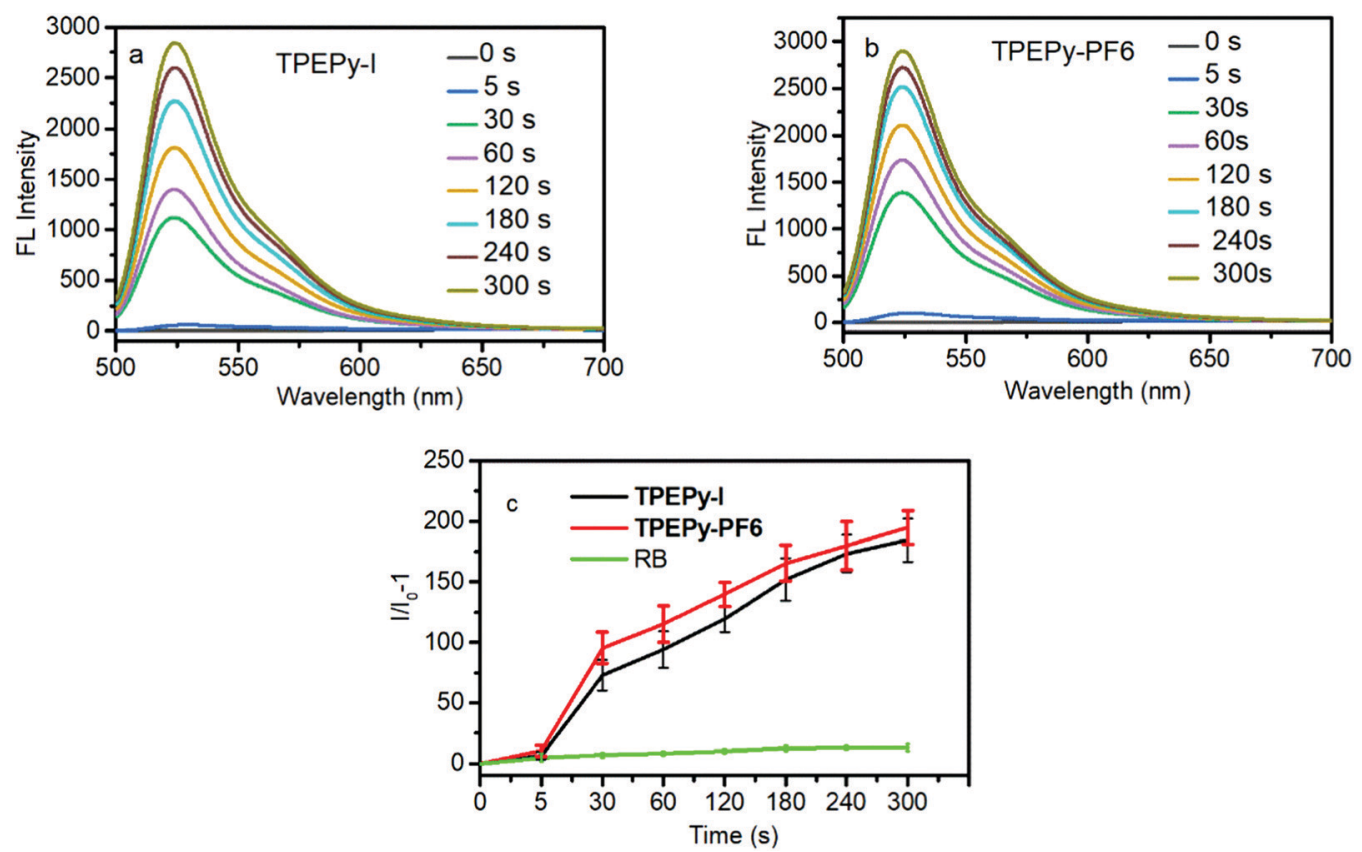

Fig. 1 Change of PL intensity at $525 \mathrm{~nm}$ of $\mathrm{H}_{2}$ DCF-DA in the presence or absence of (a) TPEPy-I (10 $\left.\mu \mathrm{M}\right)$ and (b) TPEPy-PF6 (10 $\left.\mu \mathrm{M}\right)$. (c) The fluorescence enhancement ratio of $525 \mathrm{~nm}$ of $\mathrm{H}_{2}$ DCF-DA in the presence of the two AlEgens or RB upon light irradiation for different time durations.

increasing irradiation time duration. The $525 \mathrm{~nm}$ emission reaches its maximum value after 5 min activation with a 193-fold fluorescence enhancement, suggesting that the two AIEgens have an extremely high capability for ROS generation. Such a change, however, was not observed for each AIEgen or $\mathrm{H}_{2}$ DCF-DA alone under the same conditions.

It is well known that many types of ROS including type I (radicals or radical ions such as $\mathrm{O}_{2}{ }^{\bullet-}, \mathrm{OH}^{\bullet}$, and $\mathrm{O}_{2}{ }^{2-}$ ) and type II $\left({ }^{1} \mathrm{O}_{2}\right)$ are involved in the PDT process. ${ }^{24}{ }^{1} \mathrm{O}_{2}$ is generally identified as the primary species for PDT due to its higher diffusion and stronger interaction with tissues than free radicals. ${ }^{25}$ Thus, we measured ${ }^{1} \mathrm{O}_{2}$ production by TPEPy-I and TPEPy-PF6 upon white light irradiation using 9,10-anthracenediylbis(methylene)dimalonic acid (ABDA) as a ${ }^{1} \mathrm{O}_{2}$ probe. ABDA can selectively undergo oxidation by ${ }^{1} \mathrm{O}_{2}$ to yield endoperoxid, resulting in a corresponding decrease in its absorbance. As shown in Fig. 2a and b, the characteristic absorption peaks of ABDA decrease promptly with prolonged light irradiation. After 3 minutes exposure to white light, only 40\% ABDA degraded in the presence of Rose Bengal (the most widely used PS in PDT), compared to $87 \%$ and $85 \%$ ABDA consumption in the presence of TPEPy-I and TPEPy-PF6 aggregates, respectively (Fig. 2c and d). When Rose Bengal (RB) was employed as the standard PS (0.75 in water), the ${ }^{1} \mathrm{O}_{2}$ quantum yields of TPEPy-I and TPEPy-PF6 were calculated to be 0.89 and 0.93 , respectively, indicating a high ${ }^{1} \mathrm{O}_{2}$ generation efficiency by the two AIEgens (Fig. S12, ESI $\dagger$ ). Their ${ }^{1} \mathrm{O}_{2}$ efficiencies are even higher than those of clinically used PSs such as Photofrin (0.28) or Laserphyrin (0.48). ${ }^{26}$

We further detected ROS formation by TPEPy-I and TPEPy-PF6 inside HeLa cells upon white-light activation using $\mathrm{H}_{2}$ DCF-DA. As shown in Fig. S13 (ESI $\dagger$ ), an obvious green fluorescence signal was observed inside the cells from $\mathrm{H}_{2}$ DCF-DA when it was incubated into the cells with either of the two AIEgens, indicating the formation of ROS inside the cells. The superior ROS and ${ }^{1} \mathrm{O}_{2}$ generation efficacy suggests that the two AIEgens are ideal PS candidates for PDT applications.

\subsection{TD-DFT calculation}

As we know, there are inherent relationships between ROS generation and intersystem crossing (ISC). ISC between the singlet and triplet excited states of molecules is a prerequisite for ${ }^{1} \mathrm{O}_{2}$ generation. It has been recognized that small singlettriplet gaps appreciably boost ISC rates. The ISC rate $\left(k_{\mathrm{ISC}}\right)$ can be estimated from an empirical formula based on perturbation theory: $k_{\mathrm{ISC}} \propto\left|\left\langle\mathrm{S}_{m}\left|\mathrm{H}_{\mathrm{SO}}\right| \mathrm{T}_{n}\right\rangle\right|^{2} /\left(\Delta E_{\mathrm{S}-\mathrm{T}}\right)^{2} . \mathrm{H}_{\mathrm{SO}}$ is the spin-orbit perturbation Hamiltonian and $\left\langle\mathrm{S}_{m}\left|\mathrm{H}_{\mathrm{SO}}\right| \mathrm{T}_{n}\right\rangle$ is the spin-orbit matrix element (SOCME) between the $m$ th singlet excited state and the adjacent $n$th triplet excited state. $\Delta E_{\mathrm{S}-\mathrm{T}}$ is the singlettriplet energy gap of the ISC channel of interest, which can play a crucial role in enhancing the ISC rate, as well as the high ${ }^{1} \mathrm{O}_{2}$ generation efficiency. To gain a mechanistic understanding of the extremely high ${ }^{1} \mathrm{O}_{2}$ quantum yield of the two AIEgens, we carried out time-dependent density functional theory (TD-DFT) calculations to optimize their excited states structures and calculate $\Delta E_{\mathrm{S}-\mathrm{T}}$ using the Gaussian 09 program and compute SOCME under scalar relativistic effects at the level of the zeroorder regular approximation (ZORA) as implemented in the ORCA 4.2.1 program (more detail is provided in the ESI $\dagger$ ). The conductor-like polarizable continuum model (C-PCM) for water was employed to account for the implicit solvent effect of water in experiments.

We firstly found through natural transition orbital (NTO) analysis (Fig. S14, ESI $\dagger$ ) that the lowest singlet excited state $\left(\mathrm{S}_{1}\right)$ of TPEPy $^{+}$is mainly a local transition (LT) in the acceptor with a minor participation in the donor. It has a strong light adsorption, 

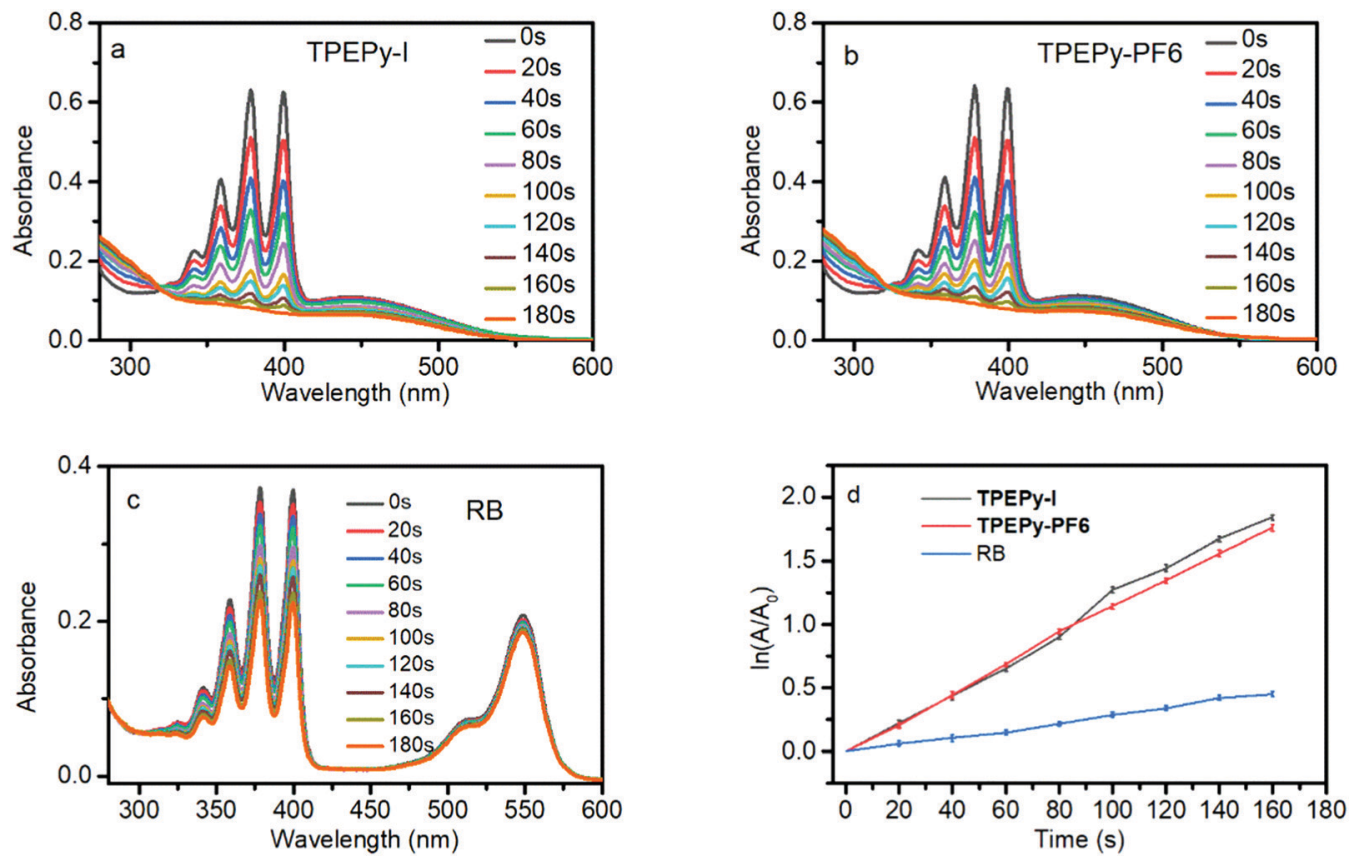

Fig. 2 (a) UV-vis absorption spectra of ABDA $\left(100 \times 10^{-6} \mathrm{M}\right)$ in (a) TPEPy-I and (b) TPEPy-PF6 solutions $\left(5 \times 10^{-6} \mathrm{M}\right)$ irradiated for different durations with white light irradiation $\left(30 \mathrm{~mW} \mathrm{~cm}^{-2}\right.$ ). (c) UV-vis absorption spectra of ABDA $\left(100 \times 10^{-6} \mathrm{M}\right)$ irradiated for different durations with white light irradiation (30 $\left.\mathrm{mW}_{\mathrm{cm}}{ }^{-2}\right)$. (d) Absorbance of ABDA at $380 \mathrm{~nm}$ in TPEPy-I, TPEPy-PF6 and Rose Bengal (RB) aqueous solutions for different durations with white light irradiation.

indicated by an oscillator strength $(f)$ of 1.94 . Similarly, the lowest spin-forbidden triplet state $\left(\mathrm{T}_{1}\right)$ is also a $\mathrm{LT}$ in the acceptor part. Considering Kasha's rule, we assume that all types of transitions (fluorescence, thermal relaxation, and ISC) after excitation of this molecule will start from $\mathrm{S}_{1}$. We found that the third lowest triplet state $\left(T_{3}\right)$ is the most adjacent triplet state to $S_{1}$. They can form effective ISC channels, thanks to the small $\Delta E_{\mathrm{S}-\mathrm{T}}\left(\mathrm{S}_{1} \rightarrow \mathrm{T}_{3}\right.$ : $-0.22 \mathrm{eV})$, albeit the SOCME is small $\left(\mathrm{S}_{1} \rightarrow \mathrm{T}_{3}: 0.33 \mathrm{~cm}^{-1}\right)$. The intrinsically small spin-orbit coupling is typical for pure organic chromophores. To further strengthen the ISC, we replace $\mathrm{PF}_{6}{ }^{-}$ with an iodide ion $\left(\mathrm{I}^{-}\right)$, based on well-accepted experience that halogen atoms can effectively boost ISC of organic molecules because of their prominent relativistic effect. $\mathrm{I}^{-}$and TPEPy $^{+}$form a weakly bound complex, with $\mathrm{I}^{-}$being $c a$. $3.7 \AA$ away from the center of the pyridine ring. As clearly shown in Table 1 , the introduction of $\mathrm{I}^{-}$greatly increases the SOCME of the $\mathrm{S}_{1} \rightarrow \mathrm{T}_{3}$ ISC channel by two orders of magnitude. This is because of the contribution of the $5 \mathrm{p}$ orbital of $\mathrm{I}$ to $\mathrm{S}_{1}$ and $\mathrm{T}_{3}$ (Fig. S14, ESI $\dagger$ ), which enables efficient spin-orbit coupling. The reduction of $\Delta E_{\mathrm{S}-\mathrm{T}}$ also helps to boost the ISC from $\mathrm{S}_{1}$ to $\mathrm{T}_{3}$. Furthermore, the $\mathrm{T}_{1}-\mathrm{S}_{0}$ gaps of both TPEPy-PF ${ }_{6}$ and TPEPy-I are sufficiently large $(1.17 \mathrm{eV}$ and $1.62 \mathrm{eV})$ to supply the energy needed to activate ${ }^{3} \mathrm{O}_{2}$, because previous studies revealed that the molecule should have a $\mathrm{T}_{1}$ state with an energy higher than $0.98 \mathrm{eV}$ to convert ground-state molecular oxygen into excited state singlet oxygen. ${ }^{27}$ Therefore, compared to $\mathbf{T P E P y}-\mathbf{P F}_{6}$, $\mathbf{T P E P y}$-I can more easily generate a triplet state that is a prerequisite for ${ }^{3} \mathrm{O}_{2}$ sensitization to produce ${ }^{1} \mathrm{O}_{2}$.

\subsection{Mitochondria-specific targeting bioimaging}

The intracellular distribution of TPEPy-I and TPEPy-PF6 in living HeLa cells was studied by using confocal laser scanning
Table 1 The singlet-triplet energy gap $\left(\Delta E_{S-T}\right)$ between $S_{1}$ and $T_{1}$ through $\mathrm{T}_{3}$ and their corresponding spin-orbit coupling matrix elements (SOCMEs) for TPEPy-PF 6 and TPEPy-I

\begin{tabular}{llcc}
\hline Species & Transition & $\Delta E(\mathrm{~s}-\mathrm{T}) / \mathrm{eV}$ & ${\text { SOCME } / \mathrm{cm}^{-1}}^{-}$ \\
\hline TPEPy $^{+}$ & $\mathrm{S}_{1} \rightarrow \mathrm{T}_{1}$ & 1.27 & 0.04 \\
& $\mathrm{~S}_{1} \rightarrow \mathrm{T}_{2}$ & 0.76 & 0.32 \\
& $\mathrm{~S}_{1} \rightarrow \mathrm{T}_{3}$ & -0.22 & 0.33 \\
TPEPy-I & $\mathrm{S}_{0} \rightarrow \mathrm{T}_{1}$ & 1.17 & 0.19 \\
& $\mathrm{~S}_{1} \rightarrow \mathrm{T}_{1}$ & 1.30 & 5.45 \\
& $\mathrm{~S}_{1} \rightarrow \mathrm{T}_{2}$ & 0.47 & 5.21 \\
& $\mathrm{~S}_{1} \rightarrow \mathrm{T}_{3}$ & -0.03 & 33.18 \\
& $\mathrm{~S}_{0} \rightarrow \mathrm{T}_{1}$ & 1.62 & 0.98
\end{tabular}

Note: for simplicity, TPEPy-PF $_{6}$ is modeled by its cation part, i.e. TPEPy $^{+}$, in TD-DFT calculations. All energies are computed using the $\omega$ B97XD functional in conjunction with the TZVP basis set for all light elements and SDD pseudopotential for iodine. The implicit solvent effect of water is treated by the C-PCM solvation model.

microscopy (CLSM). After treatment with TPEPy-I and TPEPyPF6 for $1 \mathrm{~h}$, HeLa cells exhibited a strong red fluorescence in the cytoplasm, suggesting a fast permeability of AIEgens into living cells. To realize the specificity of the AIEgens for cell imaging, co-localization experiments were then carried out with MitoTracker Deep Red, which is a commercial probe for mitochondrial imaging. It is interesting to find that TPEPy-I and TPEPy-PF6 can specifically stain the mitochondria. The perfect overlapping of their images with that of MitoTracker Deep Red gives rise to high overlap coefficiencies (0.97) (Fig. 3), indicating their superior specificities for staining mitochondria. The excellent mitochondria-targeting specificity could be attributed to the suitable lipophilicity and cationic property of AIEgens. ${ }^{28}$ 

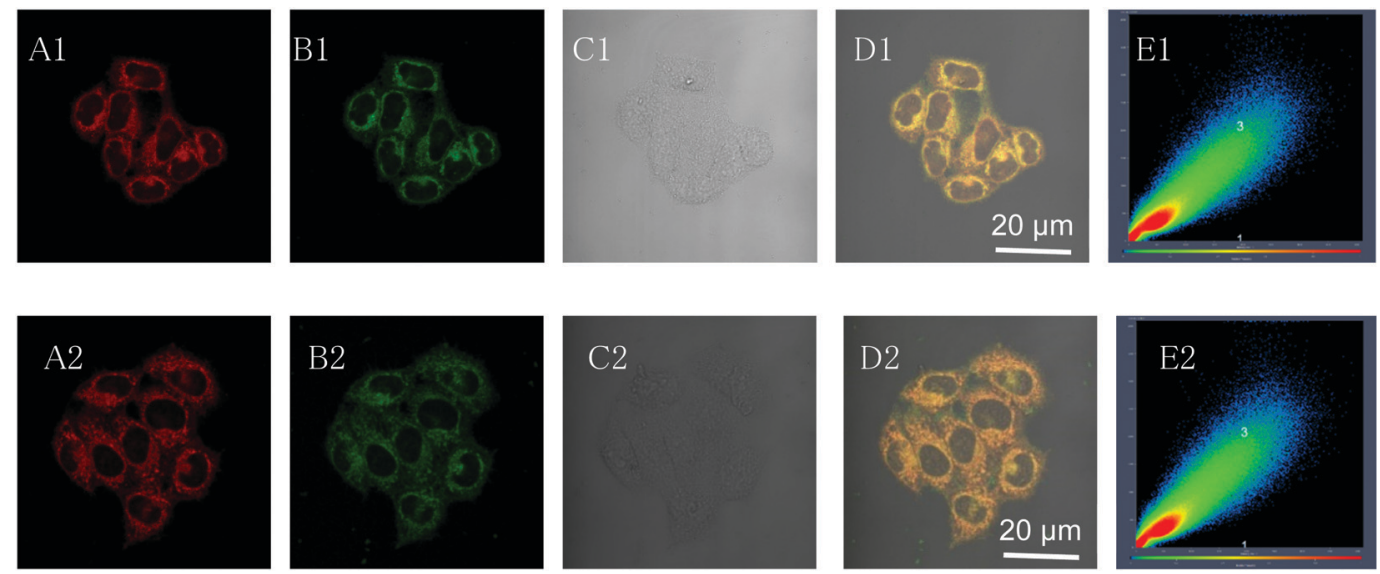

Fig. 3 Co-localization imaging of HeLa cells stained with MitoTracker Deep Red (MTDR), and TPEPy-I and TPEPy-PF6. (A and B) Confocal images of HeLa cells stained with (A1) TPEPy-I, (A2) TPEPy-PF6, and (B1 and B2) pseudo-color of MitoTracker Deep Red. (C1 and C2) Bright field. (D1 and D2) Merged images of panels (A)-(C). (E1 and E2) Scatter plot indicating the correction coefficient between panel (A) and (B). $\lambda_{\text {ex: }} 405 \mathrm{~nm}$. Concentration: $10 \mu \mathrm{M}$ (TPEPy-I and TPEPy-PF6), $50 \mathrm{nM}$ (MTDR). MitoTracker Red, $\lambda_{\mathrm{ex}}=543 \mathrm{~nm}, \lambda_{\mathrm{em}}=548-683 \mathrm{~nm}$, TPEPy-I and TPEPy-PF6, $\lambda_{\mathrm{ex}}=488 \mathrm{~nm}$, $\lambda_{\mathrm{em}}=600-650 \mathrm{~nm}$.

\subsection{PDT in cancer cell ablation}

TPEPy-I and TPEPy-PF6 exhibit a negligible dark cytotoxicity at concentrations up to $50 \mu \mathrm{M}$ with $90 \%$ viability of HeLa cells (Fig. S15, ESI $\dagger$ ), which means they have a good biocompatibility.

In contrast with their low dark cytotoxicity, TPEPy-I and TPEPy-PF6 exhibit a high phototoxicity (Fig. 4). For example, the cell viabilities are found to be $88.8 \% v s .93 .6 \%, 70.4 \% v s$. $92.6 \%$, and $58.2 \%$ vs. $66.6 \%$ at $0.05,0.1$ and $0.5 \mu \mathrm{M}$ for TPEPy-I and TPEPy-PF6, respectively. This indicates that TPEPy-I is more efficient in cancer cell ablation than TPEPy-PF6 at low concentrations less than $0.5 \mu \mathrm{M}$. As the concentration of the two AIEgens reaches $1 \mu \mathrm{M}$, there is no actual difference between them; both are effective in cancer cell ablation with the cell viability lower than $5 \%$ upon white light irradiation. The dependence of cell viability on the PS dose is in line with the characteristics of PDT. The high potency at low concentration indicates that TPEPy-I and TPEPy-PF6 outperform most conventional PSs, such as porphyrin, chlorin, BODIPY, or their respective derivatives. ${ }^{2}$ As we know, mitochondrion is the main organelle targeted by PDT, and the mitochondria-specific targeting capability ${ }^{29}$ and rapid ${ }^{1} \mathrm{O}_{2}$ generation of TPEPy-I and TPEPy-PF6 make them ideal for photodynamic applications, which cause damage in situ to exert excellent therapeutic efficiency.

\subsection{Observation of cell death and morphological changes during PDT}

The live- and dead-cell staining experiments were used to further justify the photodynamic efficacy of TPEPy-I and TPEPy-PF6. The representative images are presented in Fig. 5 and Fig. S16 (ESI $\dagger$ ), where live and dead cells were stained by calcein AM (green fluorescence) and propidium iodide (red fluorescence), respectively. Under white light irradiation for different time durations, the population of dead cells, as indicated by red fluorescence, increased while the live cell population as indicated by green emission decreased. More cells were destroyed in both cases with the increase of the irradiation duration. After white light irradiation for $5 \mathrm{~min}$, almost all HeLa cells were killed in both cases, indicating the high PDT efficacy of TPEPy-I and TPEPy-PF6.

In addition, we found that laser irradiation induced a dramatic change in the cell morphology. Interestingly, bubble generation was present during the CLSM observations, where calcein AM was used to detect cell survival for labeling.
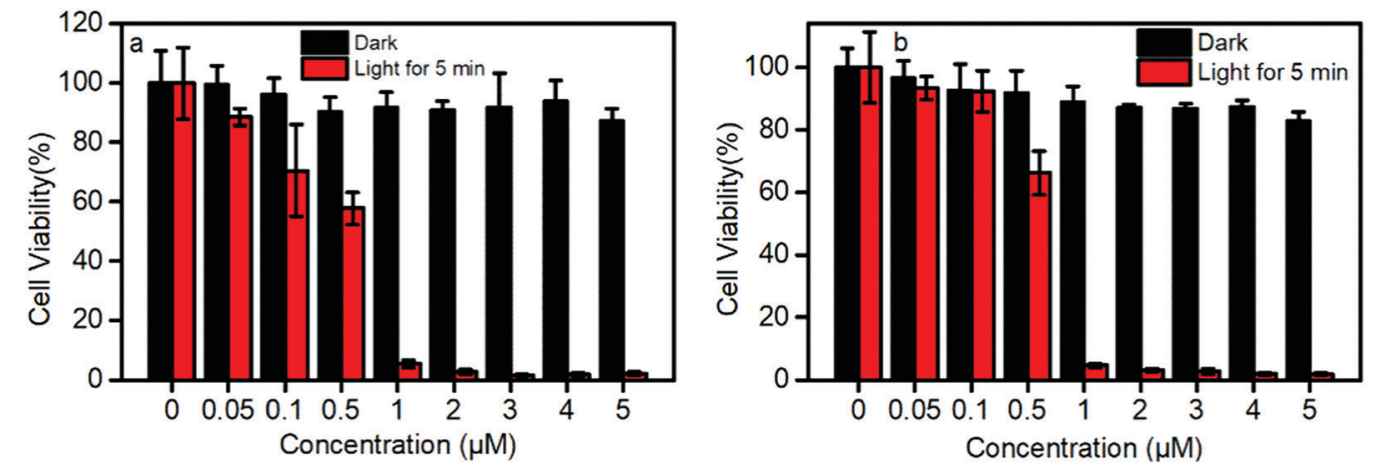

Fig. 4 Effect of (a) TPEPy-I and (b) TPEPy-PF6 with and without room-light irradiation on cell proliferation of HeLa cells evaluated by the MTT assay. Light power: $30 \mathrm{~mW} \mathrm{~cm}^{-2}$. 
PI Calcein-AM Bright field Merged
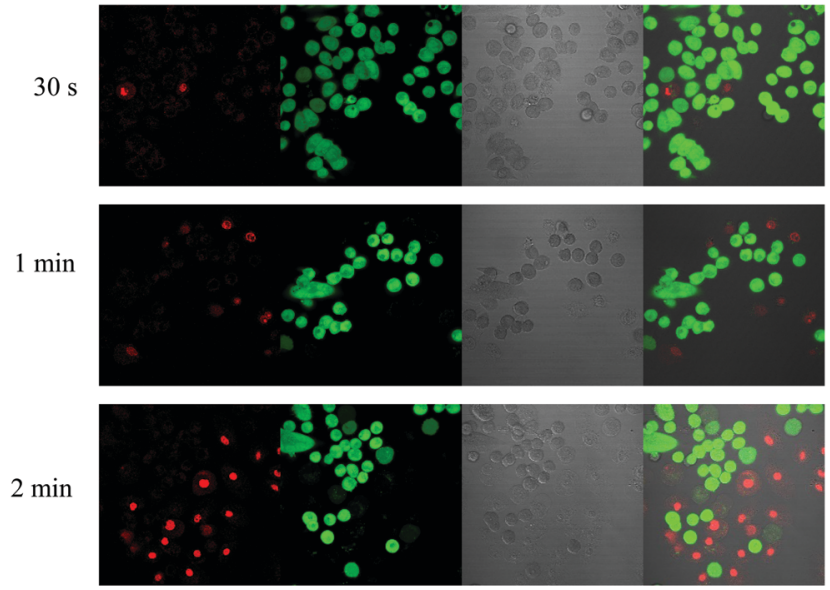

$3 \mathrm{~min}$
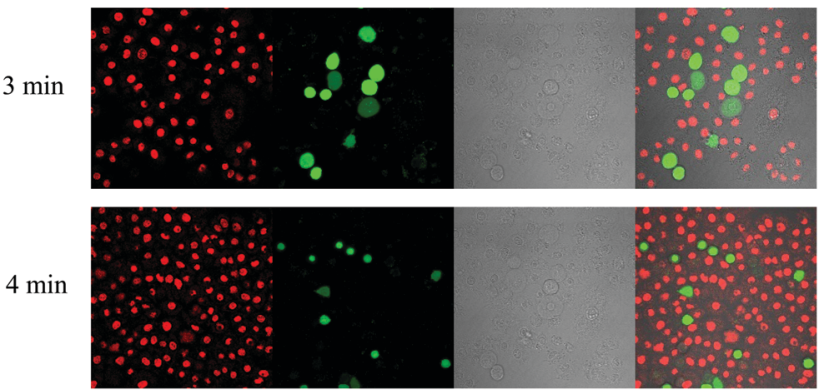

$5 \mathrm{~min}$

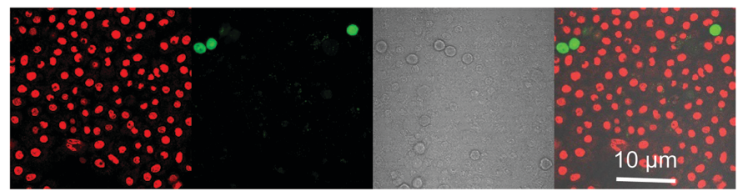

Fig. 5 Live/dead staining of TPEPy-I $(10 \mu \mathrm{M})$ treated HeLa cells with light irradiation for $30 \mathrm{~s}, 1 \mathrm{~min}, 2 \mathrm{~min}, 3 \mathrm{~min}, 4 \mathrm{~min}$ and $5 \mathrm{~min}$. The live cells were stained by calcein-AM (green), whereas dead cells were stained by PI (red).

Before irradiation, regular and normal cell morphology was observed. However, upon laser stimulation, swelling and blebbing appeared on the cells. More bubbles formed with increasing laser duration (Fig. 6a). As we know, cell morphological changes, such as blebbing, are a sign of cell death. ${ }^{30}$ When the cells were irradiated by laser for desired time (20 scanning cycles) and then left for 5 min for PI staining, the red emission from PI in the cell nuclei was obviously present due to the PI intercalating with the DNA from the nuclei. These changes can be attributed to the fact that the ROS generated from the AIEgen considerably disrupted the rigidity and permeability of the plasma membrane, causing cancer cell apoptosis and necrosis. The bright field images also confirmed that the shape of the cell changed dramatically after HeLa cells were stained by AIEgens under white light irradiation (Fig. 6b). The shrinkage and fusion of cell walls as well as the appearance of multiple protuberances were found, providing direct and strong evidence for the toxicity on HeLa cells.

\subsection{Bacterial elimination}

Since TPEPy-I and TPEPy-PF6 are positively charged, it is expected that they can target bacteria through electrostatic (a)

1 scan

10 scans

20 scans
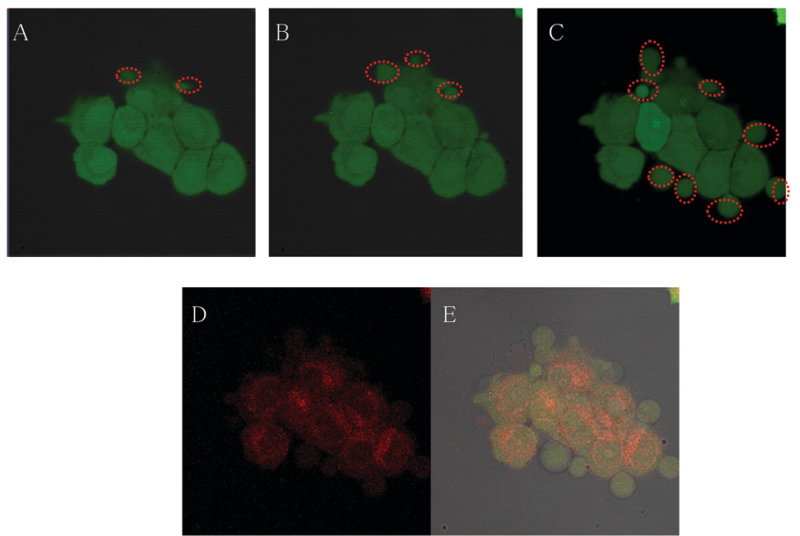

Dark

Light

(b)
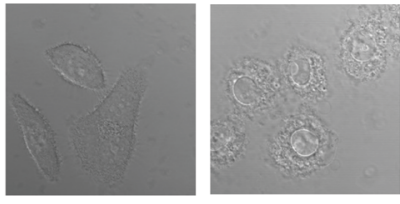

TPEPy-PF6
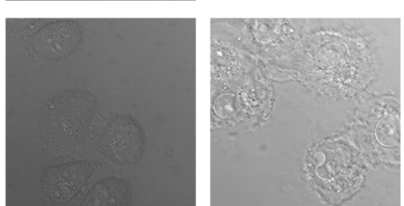

Fig. 6 (a) The CLSM images of TPEPy-I-pretreated HeLa cells stained by calcein-AM after (A) 1, (B) 10, and (C) 20 scans. (D) The CLSM images of TPEPy-I-pretreated HeLa cells stained by PI after 20 scans. (E) Merged image of (C and D). (b) The bright field images of irradiated TPEPy-I and TPEPy-PF6-pretreated living HeLa cells. [TPEPy-I] and [TPEPy-PF6] = $10 \mu \mathrm{M}$, irradiation for $5 \mathrm{~min}$.

interactions. ${ }^{31}$ The bacterial staining performance of the two AIEgens on various bacteria was observed by using CLSM. Gram-positive $S$. aureus and Gram-negative $E$. coli were selected as representatives, because they are negatively charged and facilitate the positively charged AIEgens to stain bacteria. The bright red fluorescence in $S$. aureus incubated with TPEPy-I and TPEPy-PF6 was readily observed with high contrast relative to the background (Fig. S17, ESI $\dagger$ ), indicating that both AIEgens can bind to Gram-positive bacteria efficiently. However, the two AIEgens failed to stain $E$. coli, where scarcely any fluorescence signals could be detected under CLSM (Fig. S18a, ESI $\dagger$ ). The selective imaging behavior of Gram-positive bacteria verses Gram-negative bacteria could be ascribed to their differences in surface structures and chemical components.

As shown in Fig. S18b (ESI $\dagger$ ), $\mathrm{G}^{+}$bacteria only have a cytoplasmic membrane covered by a loose and poriferous cell wall, and a crosslinked and thick peptidoglycan layer about $20-80 \mathrm{~nm}$ in size with acidic residues in the outer walls. In contrast, $\mathrm{G}^{-}$bacteria have a thinner peptidoglycan layer, which is embedded in the phospholipid bilayer. Meanwhile, $\mathrm{G}^{-}$bacteria possess an additional outer membrane, which performs a barrier function. ${ }^{32}$ These differences in the two types of pathogens allow AIEgens to penetrate their cell membrane and thus localize in them in 
different microenvironments. No fluorescence signals detected under CLSM were observed for E. coli, while S. aureus presented bright red fluorescence with high labeling efficiency, indicating the distinct binding affinities of the two AIEgens to the two pathogens. Lacking the protection of an outer membrane, TPEPy-I and TPEPy-PF6 can readily penetrate the cell membrane and enter the inside of $S$. aureus. The two AIEgens are restricted effectively by the internal environment, which turns on their emission. For E. coli, it is possible that the outer membrane (phospholipid layer) inhibits the insertion of the two AIEgens or the inserted part is easily removed due to weak interaction between $E$. coli and the two AIEgens. Several references reported selective imaging of $\mathrm{G}^{+}$over $\mathrm{G}^{-}$with AIEgens. ${ }^{33}$ The bacterial imaging results indicate that TPEPy-I and TPEPy-PF6 have the potential to selectively recognize Gram-positive bacteria over Gram-negative ones.

Next, we examined the antibacterial activity of TPEPy-I and TPEPy-PF6 upon white light irradiation. The standard plate colony-counting method was used to determine the percentage of live bacteria. The two AIEgens display a dose-dependent antibacterial efficiency toward both $S$. aureus and E. coli. Regarding the ability of TPEPy-I and TPEPy-PF6 to kill $S$. aureus, the inhibition percentage is more than $92 \%$ and $97 \%$ at $0.5 \mu \mathrm{M}$ AIEgens, respectively (Fig. 7a and b). When the concentration of the two
AIEgens reached $1 \mu \mathrm{M}$ (Fig. 7c), the inhibition percentage reached nearly $100 \%$. For commercial PSs, the concentration of Toluidine blue O used to kill more than $99.0 \%$ S. aureus is up to $80 \mu \mathrm{M} .{ }^{34}$ For chlorin e6 (Ce6), the concentration required to kill $99.9 \%$ of S. aureus is $40 \mu \mathrm{M} .^{35}$ This comparison strongly suggests that TPEPy-I and TPEPy-PF6 are excellent antibacterial PDT agents for $S$. aureus.

Meanwhile, TPEPy-I exhibits a better antibacterial activity toward E. coli than TPEPy-PF6 at low concentrations less than $1 \mu \mathrm{M}$. About $97 \% E$. coli was eliminated at the concentration of $5 \mu \mathrm{M}$ AIEgens (Fig. 8). The killing effect of TPEPy-I and TPEPyPF6 on $S$. aureus is more efficient than that on E. coli because Gram-negative bacteria have an additional protecting layer in the outer membrane. Similar findings have been reported in previous studies. ${ }^{36}$ Photographs of bacteria cultured on agar plates further confirmed the results mentioned above. Almost no bacterial colony was observed on the agar for S. aureus and E. coli in the presence of 1 and $5 \mu \mathrm{M}$ AIEgens, respectively, which showed better antibacterial activity than that of commercial Ce6 (Fig. 8c).

Some control experiments were carried out. In the dark or AIEgens alone or light alone, no obvious drop in the survival rate of bacteria was found, which suggests that the potent antimicrobial activity is entirely a consequence of the intrinsic ROS generation induced by AIEgens and white-light irradiation
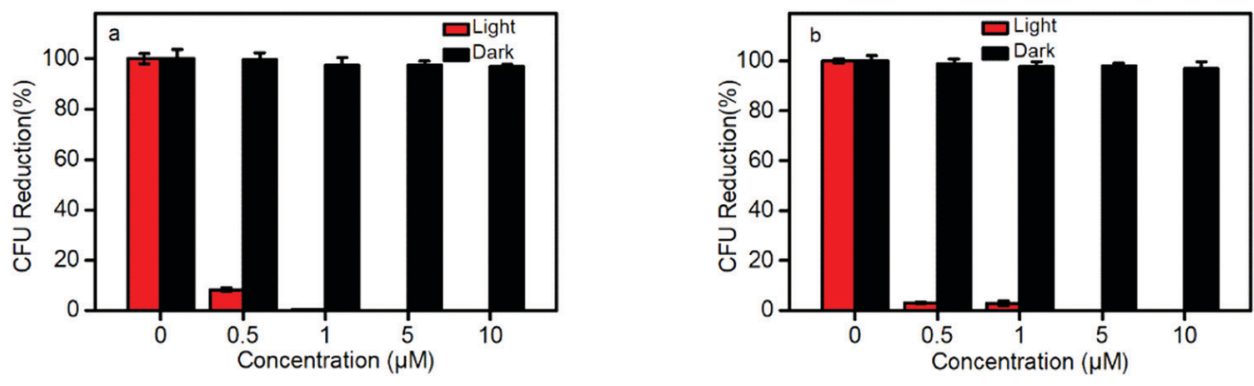

C

Dark

$0.5 \mu \mathrm{M}$

$1.0 \mu \mathrm{M}$

$5 \mu \mathrm{M}$

$10 \mu \mathrm{M}$

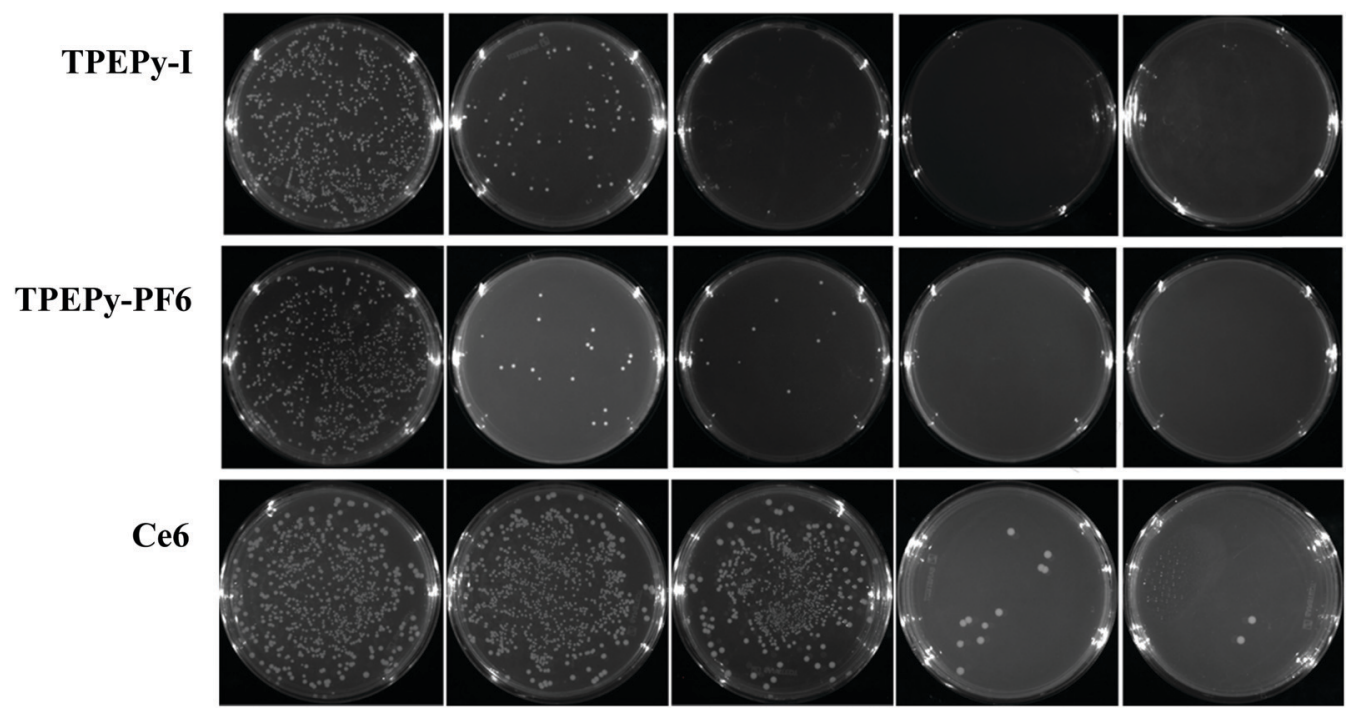

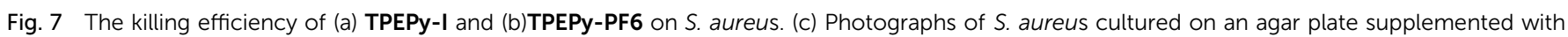
TPEPy-I, TPEPy-PF6 and Ce6 at different concentrations. 

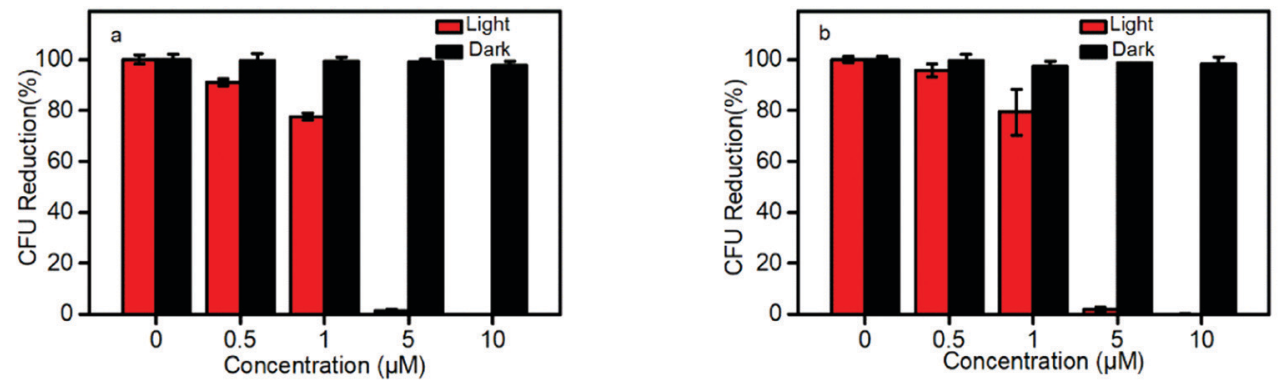

c

Dark

$0.5 \mu \mathrm{M}$

$1.0 \mu \mathrm{M}$

$5 \mu \mathrm{M}$

$10 \mu \mathrm{M}$

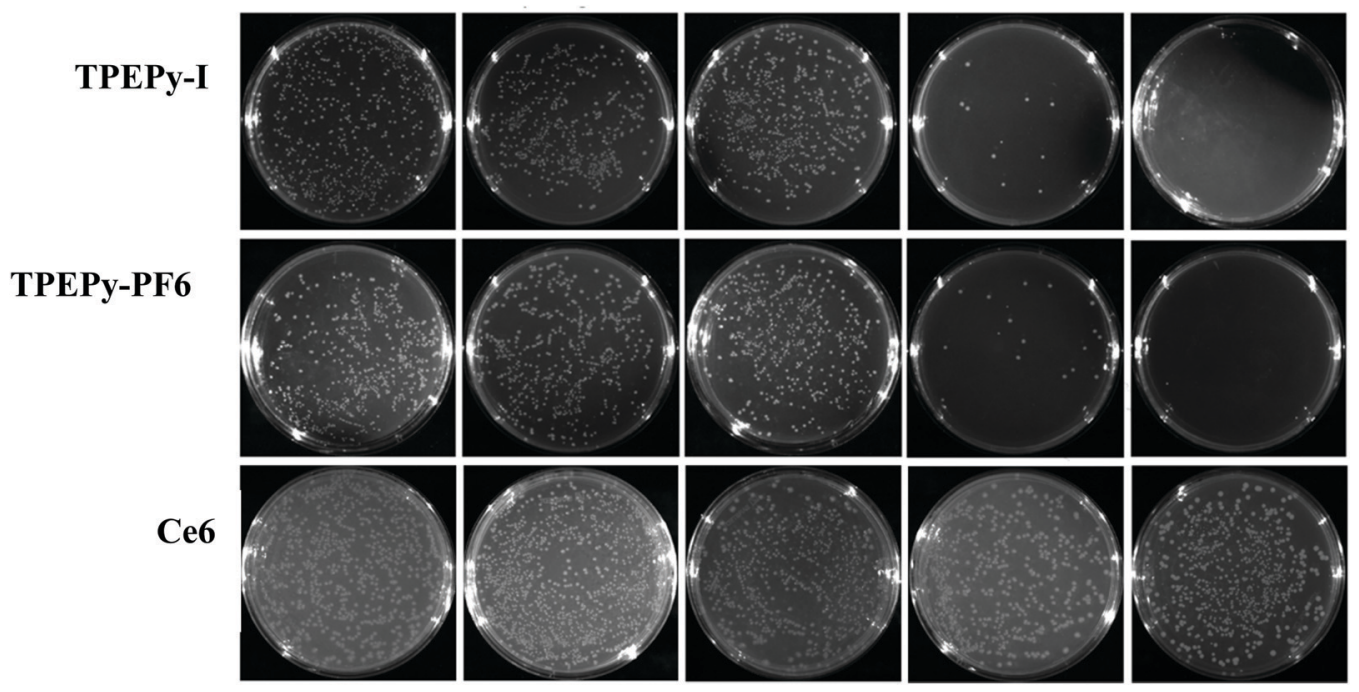

Fig. 8 The killing efficiency of (a) TPEPy-I and (b) TPEPy-PF6 on E. coli. (c) Photographs of E. coli cultured on an agar plate supplemented with TPEPy-I, TPEPy-PF6 and Ce6 at different concentrations.

(Fig. S19, ESI $\dagger$ ). All results indicate that TPEPy-I and TPEPy-PF6 possess excellent antibacterial activity towards both Grampositive and Gram-negative bacteria at low PS concentrations compared to reported data (Table S1, ESI $\dagger$ ), suggesting that they are excellent broad-spectrum antibacterial agents.

PDT can target both external and internal structures of bacteria, and it does not really require the PSs to enter the bacteria, thus the sterilization mechanism of PDT is different from traditional antibiotics. It has been proposed that the cationic PS can penetrate the outer membrane of Gram-negative bacteria by the "self-promoted uptake pathway" in which the divalent metal cations $\mathrm{Ca}^{2+}$ and $\mathrm{Mg}^{2+}$ are gradually displaced by the PS, and the lipopolysaccharide in the outer membrane permeability barrier is destabilized. ${ }^{37}$ In our case, the two AIEgens exhibited remarkable ${ }^{1} \mathrm{O}_{2}$ generation ability (up 93\%) under illumination of white light. So, the proposed molecules can provide an efficient bacterial inactivation route toward E. coli. Some white light triggered PDT methods using AIEgens with highly efficient cancer cell ablation and bacterial inactivation have been reported. ${ }^{38}$

\subsection{The mechanism of cancer cell ablation and bacterial elimination}

From the results discussed above, TPEPy-I is more efficient in cancer cell ablation and bacterial elimination than TPEPy-PF6.
The proposed mechanism can be ascribed to several possibilities. First, TPEPy-I may produce more triplet states in favor of ${ }^{1} \mathrm{O}_{2}$ formation from the TD-DFT calculations. Second, more ROS can be generated through iodide anions involved a type-I electrontransfer photochemical mechanism. Third, the iodide anion can be oxidized to molecular iodine $\mathrm{I}_{2}$ or $\mathrm{I}_{3}{ }^{-}$by both type-I and type-II ROS. $^{39}$ The higher generation of toxic species, such as $\mathrm{H}_{2} \mathrm{O}_{2}$, triiodide ions, and singlet oxygen from TPEPy-I may be the major killing mechanism for its enhanced cancer cell ablation and bacterial elimination activity. Some groups have reported similar results that the combination of triiodide with $\mathrm{H}_{2} \mathrm{O}_{2}$ can promote cell and microbial killing. ${ }^{40}$

The decrease of mitochondrial membrane potential (MMP) is a crucial indicator to assess the dysfunction of mitochondria and it plays an important role in the release of the pro-apoptotic proteins to trigger caspase activation and cell apoptosis. ${ }^{41}$ The change of mitochondrial membrane potential was determined using JC-10 dye as the indicator, which tends to aggregate with red fluorescence with a high MMP but becomes monomeric with green fluorescence with a low MMP. Therefore, the change of JC-10 fluorescence can be used to assess the status of mitochondria. As shown in Fig. S20 (ESI $\dagger$ ), under irradiation from white light, HeLa cells incubated with TPEPy-I and TPEPyPF6 display enhanced green fluorescence. The ratio of $I_{\text {green }} / I_{\text {red }}$ 
in the presence of the two AIEgens was higher than that of light alone, proving that the generated ROS by the two AIEgens injured the cellular mitochondria and resulted in the loss of the MMP.

As we know, cationic fluorescent dyes preferr to stain mitochondria in living cells through electronic interaction due to the negative charge of the mitochondrial inner membrane. ${ }^{42}$ We assume that TPEPy-I and TPEPy-PF6 could be taken up by cancer cells through endocytosis. Then, the two AIEgens selectively accumulated into the mitochondria, thereby inducing efficient mitochondrial dysfunction and intrinsic cancer cell apoptosis by PDT.

\section{Conclusions}

In summary, two NIR AIEgens (TPEPy-I and TPEPy-PF6) based on an electron push-pull framework are synthesized and characterized. Due to their AIE backbones with D-A structural units, both the AIEgens show broad absorption in the visible range, while a cationic group is introduced into the molecular design for mitochondriaspecific targeting and bacterial membrane anchoring. They can generate ROS efficiently inside cancer cells and bacteria, causing oxidative damage to the mitochondria of cancer cells and cell walls of bacteria. It was demonstrated that low concentrations of AIEgens $(1 \mu \mathrm{M})$ almost completely kill cancer cells upon white light irradiation. Moreover, benefitting from the enhanced membrane interactions and photosensitizing ability, TPEPy-I and TPEPy-PF6 exhibited efficient antibacterial capability in destroying $S$. aureus and $E$. coli at a low concentration $(0.5 \mu \mathrm{M})$. These results revealed the great potential of these AIEgens with NIR emission, a high yield of ROS generation, and low dark toxicity to serve in image guided PDT.

\section{Conflicts of interest}

There are no conflicts to declare.

\section{Acknowledgements}

The support by the China Scholarship Council (201906155012), the National Key Research and Development Program of China (2016YFA0602900), the National Natural Science Foundation of China (21772045), the Natural Science Foundation of Guangdong Province (2016A030311034, 2018B030311008) and Technology Program of Guangzhou (201904010414) is gratefully acknowledged. G. Z. Zhang is grateful to the Supercomputing Center of University of Science and Technology of China for the computing resource. W. Chen would like to acknowledge the distinguished record award in research/creative activity from the University of Texas.

\section{References}

1 B. D. Wallace, H. Wang, K. T. Lane, J. E. Scott, J. Orans, J. S. Koo, M. Venkatesh, C. Jobin, L. A. Yeh, S. Mani and M. R. Redinbo, Science, 2010, 330, 831-835.

2 (a) D. E. J. G. J. Dolmans, D. Fukumura and R. K. Jain, Nat. Rev. Cancer, 2003, 3, 380-387; (b) A. P. Castano, P. Mroz and M. R. Hamblin, Nat. Rev. Cancer, 2006, 6, 535-545; (c) M. H.
Al-Afyouni, T. N. Rohrabaugh, K. F. Al-Afyouni and C. Turro, Chem. Sci., 2018, 9, 6711-6720; (d) H. Shi, X. Ma, Q. Zhao, B. Liu, Q. Qu, Z. An, Y. Zhao and W. Huang, Adv. Funct. Mater., 2014, 24, 4823-4830; (e) D. Tao, L. Feng, Y. Chao, C. Liang, X. Song, H. Wang, K. Yang and Z. Liu, Adv. Funct. Mater., 2018, 28, 1804901; $(f)$ H. Yuan, H. Chong, B. Wang, C. Zhu, L. Liu, Q. Yang, F. Lv and S. Wang, J. Am. Chem. Soc., 2012, 134, 13184-13187; (g) Y. Li, Q. Wu, M. Kang, N. Song, D. Wang and B. Z. Tang, Biomaterials, 2020, 232, 119749; (h) M. Gao and B. Z. Tang, Coord. Chem. Rev., 2020, 402, 213076; (i) C. Sun, H. Zhang, L. Yue, S. Li, Q. Cheng and R. Wang, ACS Appl. Mater. Interfaces, 2019, 11, 22925-22931; (j) Y. Ding, S. Li, L. Liang, Q. Huang, L. Yuwen, W. Yang, R. Wang and L. Wang, ACS Appl. Mater. Interfaces, 2018, 10, 9980-9987.

3 T. J. Dougherty, C. J. Gomer, B. W. Henderson, G. Jori, D. Kessel, M. Korbelik, J. Moan and Q. Peng, J. Natl. Cancer Inst., 1998, 90, 889-905.

4 (a) X. Zhao, Y. J. Yang, Y. Yu, S. M. Guo, W. X. Wang and S. M. Zhu, Chem. Commun., 2019, 55, 13542-13545; (b) G. X. Feng and B. Liu, Acc. Chem. Res., 2018, 51, 1404-1414.

5 Z. Y. Liu, H. Zou, Z. Zhao, P. F. Zhang, G. G. Shan, T. K. Kwok. Ryan, J. W. Y. Lam, L. Zheng and B. Z. Tang, ACS Nano, 2019, 13, 11283-11293.

6 N. Alifu, X. Dong, D. Li, X. Sun, A. Zebibula, D. Zhang, G. Zhang and J. Qian, Mater. Chem. Front., 2017, 1, 1746-1753.

7 X. Sun, A. Zebibula, X. Dong, G. Zhang, D. Zhang, J. Qian and S. He, ACS Appl. Mater. Interfaces, 2018, 10, 25037-25046.

8 C. Chen, H. Qu, R. Liu and D. Ding, Adv. Mater., 2019, 1806331.

9 (a) Z. Zheng, T. Zhang, H. Liu, Y. Chen, R. T. K. Kwok, C. Ma, P. Zhang, H. H. Y. Sung, I. D. Williams, J. W. Y. Lam, K. S. Wong and B. Z. Tang, ACS Nano, 2018, 12, 8145-8159; (b) B. Gu, W. Wu, G. Xu, G. Y. F. Feng, P. H. J. Chong, J. Qu, K. T. Yong and B. Liu, Adv. Mater., 2017, 29, 1701076; (c) Y. Yang, L. Wang, H. Q. Cao, Q. Li, Y. Li, M. J. Han, H. Wang and J. B. Li, Nano Lett., 2019, 19, 1821-1826.

10 (a) J. Qi, C. Chen, D. Ding and B. Z. Tang, Adv. Healthcare Mater., 2018, 7, 1800477; (b) C. Chen, X. Ni, S. R. Jia, Y. Liang, X. L. Wu, D. L. Kong and D. Ding, Adv. Mater., 2019, 31, 1904914.

11 H. Gao, X. Zhang, C. Chen, K. Li and D. Ding, Adv. Biosyst., 2018, 2, 1800074.

12 X. Ni, X. Zhang, X. Duan, H. Zheng, X. Xue and D. Ding, Nano Lett., 2019, 19, 318-330.

13 D. Wang, M. M. S. Lee, W. Xu, G. Shan, X. Zheng, R. T. K. Kwok, J. W. Y. Lam, X. Hu and B. Z. Tang, Angew. Chem., Int. Ed., 2019, 58, 5628-5632.

14 W. Wu, D. Mao, S. Xu, S. Ji, F. Hu, D. Ding, D. Kong and B. Liu, Mater. Horiz., 2017, 4, 1110-1114.

15 F. Hu, D. Mao, K. Kenry, X. Cai, W. Wu, D. Kong and B. Liu, Angew. Chem., Int. Ed., 2018, 57, 10182-10186.

16 S. Wang, W. Wu, P. Manghnani, S. Xu, Y. Wang, C. C. Goh, L. G. Ng and B. Liu, ACS Nano, 2019, 13, 4742-4751.

17 W. B. Wu, D. Mao, F. Hu, S. D. Xu, C. Chen, C. J. Zhang, X. M. Cheng, Y. Y. Yuan, D. Ding, D. L. Kong and B. Liu, Adv. Mater., 2017, 29, 1700548. 
18 S. Xu, Y. Yuan, X. Cai, C.-J. Zhang, F. Hu, J. Liang, G. Zhang, D. Zhang and B. Liu, Chem. Sci., 2015, 6, 5824-5830.

19 (a) T. Maisch, Mini-Rev. Med. Chem., 2009, 9, 974-983; (b) H. Chen, W. Zhang, G. Zhu, J. Xie and X. Chen, Nat. Rev. Mater., 2017, 2, 1-8.

20 (a) C. Zhou, W. Xu, P. Zhang, M. Jiang, Y. Chen, R. T. K. Kwok, M. M. S. Lee, G. Shan, R. Qi, X. Zhou, J. W. Y. Lam, S. Wang and B. Z. Tang, Adv. Funct. Mater., 2019, 29, 18059864; (b) R. Hu, F. Zhou, T. Zhou, J. Shen, Z. Wang, Z. Zhao, A. Qin and B. Z. Tang, Biomaterials, 2018, 187, 47-54; (c) Y. Li, Z. Zhao, J. Zhang, R. T. K. Kwok, S. Xie, R. Tang, Y. Jia, J. Yang, L. Wang, J. W. Y. Lam, W. Zheng, X. Jiang and B. Z. Tang, Adv. Funct. Mater., 2018, 28, 180463242; (d) M. Jiang, X. Gu, R. T. K. Kwok, Y. Li, H. H. Y. Sung, X. Zheng, Y. Zhang, J. W. Y. Lam, I. D. Williams, X. Huang, K. S. Wong and B. Z. Tang, Adv. Funct. Mater., 2018, 28, 1704589; (e) E. Zhao, Y. Chen, S. Chen, H. Deng, C. Gui, C. W. T. Leung, Y. Hong, J. W. Y. Lam and B. Z. Tang, Adv. Mater., 2015, 27, 4931-4937; $(f)$ M. M. Kang, C. C. Zhou, S. M. Wu, B. R. Yu, Z. J. Zhang, N. Song, M. M. S. Lee, W. H. Xu, F. J. Xu, D. Wang, L. Wang and B. Z. Tang, J. Am. Chem. Soc., 2019, 141, 16781-16789; (g) M. M. Kang, T. K. Kwok. Ryan, J. G. Wang, H. Zhang, W. Y. Lam. Jacky, Y. Li, P. F. Zhang, H. Zou, X. G. Gu, F. Li and B. Z. Tang, J. Mater. Chem. B, 2018, 6, 3894-3903; (h) Q. Y. Li, Y. Li, T. L. Min, J. Y. Gong, L. L. Du, D. L. Phillips, J. K. Liu, J. W. Y. Lam, H. H. Y. Sung, I. D. Williams, R. T. K. Kwok, C. L. Ho, K. Li, J. G. Wang and B. Z. Tang, Angew. Chem., Int. Ed., 2020, 59, 10184-10188.

21 G. Valduga, G. Bertoloni, E. Reddi and G. Jori, J. Photochem. Photobiol., B, 1993, 21, 81-86.

22 J. Parsonnet, Environ. Health Perspect., 1995, 103, 263-268.

23 X. M. Zhen, L. Chudal, N. K. Pandey, J. Phan, X. Ran, E. Amandor, X. J. Huang, O. Johnson, Y. P. Ran, W. Chen, M. R. Hamblind and L. Y. Huang, Mater. Sci. Eng., C, 2020, 110659.

24 (a) D. E. Dolmans, D. Fukumura and R. K. Jain, Nat. Rev. Cancer, 2003, 3, 380-387; (b) J. Moan and Q. Peng, Anticancer Res., 2002, 23, 3591-3600; (c) N. Zhao, B. Wu, X. Hu and D. Xing, Biomaterials, 2017, 141, 40-49; (d) S. Xu, Y. Yuan, X. Cai, C.-J. Zhang, F. Hu, J. Liang, G. Zhang, D. Zhang and B. Liu, Chem. Sci., 2015, 6, 5824-5830.

25 O. T. Fackler and R. Grosse, J. Cell Biol., 2008, 181, 879-884.

26 (a) E. Zhao, Y. Chen, H. Wang, S. Chen, J. W. Lam, C. W. Leung, Y. Hong and B. Z. Tang, ACS Appl. Mater. Interfaces, 2015, 7, 7180-7188; (b) W. Zhang, Y. Huang, Y. Chen, E. Zhao, Y. Hong, S. Chen, J. W. Y. Lam, J. Hou and B. Z. Tang, ACS Appl. Mater. Interfaces, 2019, 11, 10567-10577; (c) G. Feng, Y. Yuan, H. Fang, R. Zhang, B. Xing, G. Zhang, D. Zhang and B. Liu, Chem. Commun., 2015, 51, 12490-12493; (d) M. Gao, Q. Hu, G. Feng, N. Tomczak, R. Liu, B. Xing, B. Z. Tang and B. Liu, Adv. Healthcare Mater., 2015, 4, 659-663.

27 (a) C. Schweitzer and R. Schmidt, Chem. Rev., 2003, 103, 1685-1757; (b) L. P. Silva, A. Núnez-Montenegro, C. M. Magalhães, P. J. O. Ferreira, D. Duarte, P. Gonzalez-Berdullas,
J. E. Rodríguez-Borges, N. Vale and J. C. G. Esteves da Silva, Eur. J. Med. Chem., 2019, 183, 111683.

28 (a) N. Li, Y. Y. Liu, Y. Li, J. B. Zhuang, R. R. Cui, Q. Gong, N. Zhao and B. Z. Tang, ACS Appl. Mater. Interfaces, 2018, 10, 24249-24257; (b) N. Zhao, S. Chen, Y. Hong and B. Z. Tang, Chem. Commun., 2015, 51, 13599-13602; (c) D. Wang, M. M. S. Lee, G. Shan, R. T. Kwok, J. W. Y. Lam, H. Su, Y. Cai and B. Z. Tang, Adv. Mater., 2018, 30, 1802105.

29 D. Wang, H. Su, R. T. K. Kwok, X. Hu, H. Zou, Q. Luo, M. M. S. Lee, W. Xu, J. W. Y. Lam and B. Z. Tang, Chem. Sci., 2018, 9, 3685-3693.

30 C. Zhu, Q. Yang, L. Liu, F. Lv, S. Li, G. Yang and S. Wang, Adv. Mater., 2011, 23, 4805-4810.

31 (a) X. Li, H. Bai, Y. Yang, J. Yoon, S. Wang and X. Zhang, Adv. Mater., 2019, 31, 1805092; (b) F. Hu, S. Xu and B. Liu, Adv. Mater., 2018, 30, 1801350; (c) H. Chen, S. L. Li, M. Wu, K. Kenry, Z. M. Huang, C. S. Lee and B. Liu, Angew. Chem., Int. Ed., 2019, 58, 1-6.

32 (a) H. Bai, H. Chen, R. Hu, M. Li, F. Lv, L. Liu and S. Wang, ACS Appl. Mater. Interfaces, 2016, 8, 31550-31557; (b) Y. Wang, T. S. Corbitt, S. D. Jett, Y. Tang, K. S. Schanze, E. Y. Chi and D. G. Whitten, Langmuir, 2012, 28, 65-70.

33 (a) C. Zhou, M. Jiang, J. Du, H. Bai, G. Shan, R. T. K. Kwok, J. H. C. Chau, J. Zhang, J. W. Y. Lam, P. Huang and B. Z. Tang, Chem. Sci., 2020, 11, 4730-4740; (b) R. Hu, F. Zhou, T. Zhou, J. Shen, Z. Wang, Z. Zhao, A. Qina and B. Z. Tang, Biomaterials, 2018, 187, 47-54; (c) M. Kang, R. T. K. Kwok, J. Wang, H. Zhang, J. W. Y. Lam, Y. Li, P. Zhang, H. Zou, X. Gu, F. Li and B. Z. Tang, J. Mater. Chem. $B, 2018,6,3894-3903$.

34 M. Wainwright and K. B. Crossley, J. Chemother., 2002, 14, 431-443.

35 (a) X. Li, H. Bai, Y. Yang, J. Yoon, S. Wang and X. Zhang, Adv. Mater., 2019, 31, 1805092; (b) F. Hu, S. Xu and B. Liu, Adv. Mater., 2018, 30, 1801350.

36 (a) M. A. Pereira, M. A. F. Faustino, J. P. C. Tome, M. G. P. M. S. Neves, A. C. Tome, J. A. S. Cavaleiro, A. Cunha and A. Almeida, Photochem. Photobiol. Sci., 2014, 13, 680-690; (b) T. Wu, A. C. McCandlish, L. S. Gronenberg, S. S. Chng, T. J. Silhavy and D. Kahne, Proc. Natl. Acad. Sci. U. S. A., 2006, 103, 11754-11759; (c) H. Ma, Y. Ma, L. Lei, W. Yin, Y. Yang, T. Wang, P. Yin, Z. Lei, M. Yang, Y. Qin and S. Zhang, ACS Sustainable Chem. Eng., 2018, 6, 15064-15071.

37 A. Minnock, D. I. Vernon, J. Schofield, J. Griffiths, J. H. Parish and S. B. Brown, Antimicrob. Agents Chemother., 2000, 44, 522-527.

38 (a) N. Zhao, P. Li, J. Zhuang, Y. Liu, Y. Xiao, R. Qin and N. Li, ACS Appl. Mater. Interfaces, 2019, 11, 11227-11237; (b) Y. Zheng, H. Lu, Z. Jiang, Y. Guan, J. Zou, X. Wang, R. Cheng and H. Gao, J. Mater. Chem. B, 2017, 5, 6277-6281.

39 (a) X. Zhen, L. Chudal, N. K. Pandey, J. Phan, X. Ran, E. Amandor, X. Huang, O. Johnson, Y. Ran, W. Chen, M. R. Hamblind and L. Huang, Mater. Sci. Eng., C, 2020, 110, 110659; (b) L. Huang, G. Szewczyk, T. Sarna and M. R. Hamblin, ACS Infect. Dis., 2017, 3, 320-328. 
40 (a) J. Mosinger and B. Mosinger, Experientia, 1995, 51, 106-109; (b) L. Y. Huang, L. Ma, W. J. Xuan, X. M. Zhen, H. Zheng, W. Chen and M. R. Hamblin, J. Biomed. Nanotechnol., 2019, 15, 2142-2148.

41 (a) B. Purushothaman, J. Choi, S. Park, J. Lee, A. A. S. Samson, S. Hong and J. M. Song, J. Mater. Chem. B, 2019, 7,
65-79; (b) T. Qi, B. Chen, Z. Wang, H. Du, D. Liu, Q. Yin, B. Liu, Q. Zhang and Y. Wang, Biomaterials, 2019, 213, 119219.

42 (a) J. Zielonka, J. Joseph, A. Sikora, M. Hardy, O. Ouari, J. Vasquez-Vivar, G. Cheng, M. Lopez and B. Kalyanaraman, Chem. Rev., 2017, 117, 10043-10120; (b) Z. Xu and L. Xu, Chem. Commun., 2016, 52, 1094-1119. 